\title{
New Perspectives for Seed Germination Testing Through Digital Imaging Technology
}

\author{
Antonio Dell'Aquila", ${ }^{*}$
}

Institute of Plant Genetics-CNR, Via G. Amendola 165/a, 70126, Bari, Italy

\begin{abstract}
A digital image of a plant seed can be regarded as a two-dimensional object which can be measured in size, shape and color density during the development stage of germination by computer image analysis technology. Seed changes its biological structure passing from a quiescent stage to a proliferating one, and any morphological variation can be associated with the corresponding variation of seed geometry and color space components. Future perspectives are addressed to design comprehensive biological models that may contribute to studying seed quality patterns possibly integrated with those proposed for other biological systems.
\end{abstract}

Keywords: Computer image analysis, seed geometry, information technology, crop seeds.

\section{INTRODUCTION}

Over the last two decades developmental biology has led to the main problem of how patterns and corresponding imaging may be linked to a combination of transcriptors and the production of signaling molecules. It is not clear how both these factors are coupled to generate a change in shape of a living organism, also because some aspects have been described qualitatively while growth and shape changes require quantitative descriptors [1]. In the case of a crop seed germination process, changes in size and shape can be studied as a complex of tissue sweling, which culminates in tissue proliferation and/or enlargement, these events resulting in different phases of water uptake as extensively studied by the pioneer work of Bewley [2]. Digital imaging and computer simulation are useful tools to integrate complex physiological, biochemical and molecular data and perform a quantitative assessment of seed germination rate, in terms of viability and vigor degree, in automated and objective manner. The application of computational techniques to the study of seed germination covers three aspects: computer-assisted image analysis systems, descriptive simulation modeling, and combined relationmodeling between morphological changes and biological processes.

A variety of computational techniques has been provided to capture digital seed images, to set the thresholds of imageobject definition, the so-called segmentation procedure, to measure image analysis parameters and establish their patterns in connection with the geometry variation of a germinating seed. These techniques also foresee humancomputer interaction, which is required to localize and visualize time-lapse image sequence and alignment, and to track a portion of the image obtained at different times [3].

*Address correspondence to this author at the Via Abate Gimma 247-70122 Bari, Italy, Tel/Fax: +39 805241026;

E-mails: dellaquilaantonio@tiscali.it, antonio.dellaquila@igv.cnr.it

${ }^{\S}$ Formerly Senior Scientist of the CNR, Italy.
Recently, a practical application of digital imaging on seed quality testing and sorting has been extensively reviewed [4]. Automated computer methods which utilise high-speed image capturing and data processing are the most advanced techniques to replace human visual inspection, usually employed in germination tests under the rules of the ISTA [5] and AOSA [6]. As a result, new hardware architectures and algorithms have been developed for high speed extraction of raw data, i.e. digital images, and numeric data, such as dimensional measurements, shape factors and color space primary density. Integration of image analysis data with those obtained by other biological systems could contribute to up-grading the 'information technology' which accounts for acquisition, recording and processing, and communication of information. This basic knowledge could also be included in the complex system of 'precision agriculture', where farmers, seed industries, seed-gene banks, seed analysts and researchers require new information networks and tools to reduce costs and environmental impacts, while enhancing and protecting plant biodiversity in the establishment of sustainable agro-ecosystems [7].

In this paper, we survey the perspectives of digital imaging technology in relation also to descriptive modeling and tracking simulation with the aim to advance this technique as a promising tool in studying the 'seed germination system'.

\section{PROGRESS IN IMAGING TECHNOLOGY APPLIED TO THE STUDY OF SEED GERMINATION AND GROWTH}

The digital seed image can be regarded as a twodimensional object placed on a Cartesian plane, and subjected to a geometric variations due to the enlargement and growth of the imbibing seed. One way of describing the development of a biological structure is by the velocity at which its points move relative to a fixed coordinate system $[1,8]$. By plotting any geometric change against time units a polynomial curve may be obtained. This resembles the triphasic pattern of water uptake [2] having two inflection points which correspond, the first to the completion of seed 
enlargement due to a rapid sweling and the second, to completion of biological processes leading to the protrusion of the radicle tip from the seed coat, that is the signal of the so-called 'visible germination'. Mathematically, this behavior can be expressed by differential equations and provides a new approach which treats the germinating seed as a continuous model, where growth involves changes in dimensions, i.e. shape and size.

This mechanistic model constitutes the basic working principle of any computer-assisted image analysis system applied to the study of seed germination and automated testing of viability and seedling growth, as a combined vigor assay. Present image analysis prototypes (Fig. 1) may be designed as follows:

1. A thermostatic chamber, which includes image capturing equipments, a support for the seed germination medium, a lighting system, and a mobile camera gantry which is capable to measure a number of samples automatically without operator aid.

2. A modular image analysis system which comprises hardware to capture digital images [e.g., flat-bed scanner or a Charged Coupled Device (CCD)-camera] and a personal computer with high processor power and physical memory to be able to quickly transfer the image to the hard disk and allow image processing and measurement. A network is also required to transfer data collected by the image analyzer to other computer systems located in other institutions for the implementation of image analysis databases.

3. The use of a specific software package which can be easily reconfigured to collect digital imaging and carry out image segmentation to define the silhouette of the seed. Other desired characteristics are those to produce new algorithms in measuring image descriptors (i.e., size metric measurements, shape numeric factors, and color medium density in pixel using the most common range color space components) and to transfer data to other software applications for graphical display (e.g., two- (2-) and three-dimensional (3-D) plotting and image sequence animation) or statistical analysis. The software of image analysis is also required to be able to create a macro in $\mathrm{C}++$ Basic macro language for an automated image and data processing and elaboration. The data obtained by a seed germination image analysis system can be used to produce 2-D seed image catalogs [http://www.seedimages.com], seed image animation simulating germination process for educational purposes [http://germimaging.ba.cnr.it], and 3-D seed surface plotting models $[4,8]$.

In the last two decades, the advent of computer-aided image acquisition coupled with fast image processing and analysis has allowed to quantify several seed morphological features. A casual perusal of inherent bibliography reveals that about 30 crop species have been tested for germination and seedling growth by automated vision machines [4]. Some of these studies where focused on the automated recording of the seed sweling process as an alternative to measurement of seed weight during the course of imbibition.
Metric descriptors of seed size changes, such as area, perimeter, length and width, or numeric factors of seed shape changes, such as roundness and aspect, were used to monitor the sequence of time-lapse captured seed images, which can represent an 'image print' of seed imbibition and growth. The technique results to be highly automated, adaptable under different imbibition conditions, such as water stress, different regimes of temperature, in both highly viable and deteriorated seeds [9]. Seed area and roundness factor have been tested as effective markers of start of germination, with the advantage of being measured on each individual seed within a seed population.
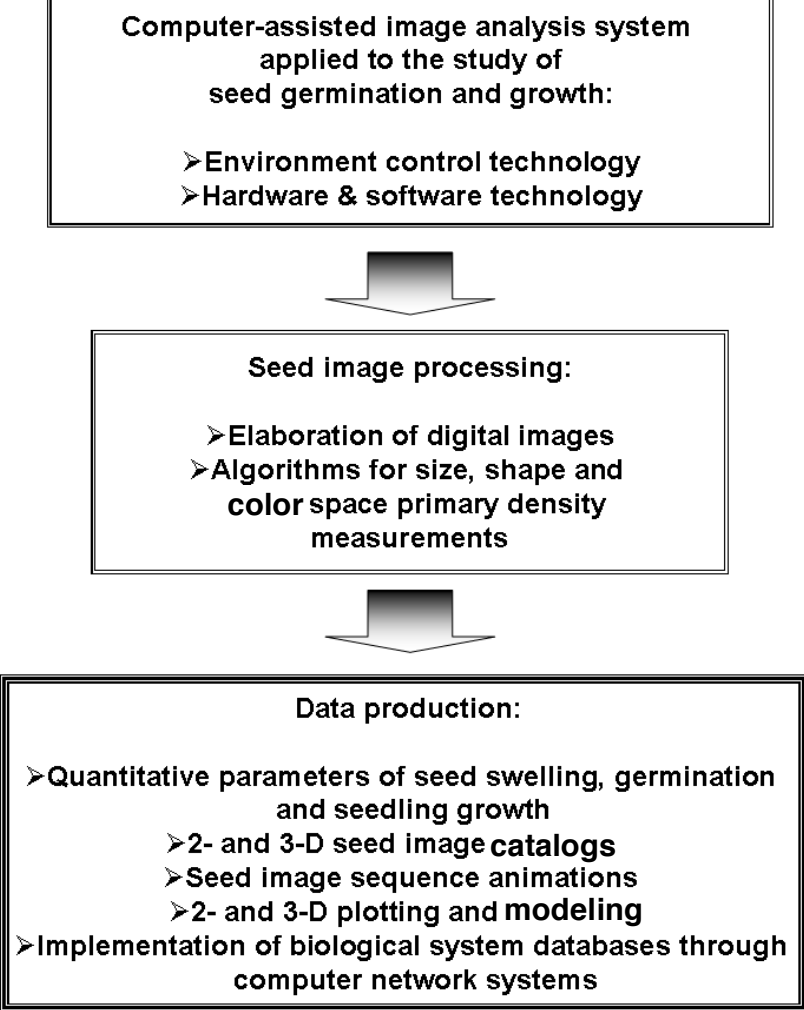

Fig. (1). Scheme of an image analysis system applied to the study of seed germination and growth and related information flow.

Image analysis could provide an innovative approach to improve the quality of data and saving working time of a seed analyst. An appropriate integration between automated germination counts with image analysis and standard methods, e.g. the inspection of normal and abnormal seedlings after completion of the germination test by a skilled analyst as requested by ISTA rules [5], could contribute for a more detailed information on vigor of a seed lot. In addition, the technique has been proved to be more useful for horticultural crop seeds having a long time germination than for those with fast germination [10], and a more precise germination parameter assessment when different temperature cycle conditions were applied during different phases of imbibition, not obtainable by a classical germination test [11]. A promising field of application of image analysis is seed vigor testing. McDonald and his colleagues first designed a special scanner contained in a metal box, used to capture images of three-day-old lettuce (Lactuca sativa L.) and soybean [Glycine max (L.) Merr.] seedlings which were processed with an appropriate software 
package $[12,13]$. The generated image analysis parameter values represented collectively a vigor index based on morphological features of the seedlings. The method was further improved in cotton (Gossypium hirsutum L.) seed [14]. The problem of overlapping seedlings, which interferes with the measurements of elongation rate, was solved by the design of a new algorithm which measures each seedling independently. It has been also demonstrated that the rate of increase in seed area, mostly due to protrusion of the radicle and its growth, may be correlated with the corresponding radicle length, as described in Brassica, radish (Raphanus sativus L.), lentil (Lens culinaris Medik.), lettuce, pepper (Capsicum annuum L.), tomato (Lycopersicon esculentum L.), and carrot (Daucus carota subsp. Sativa) seeds, providing an indirect and automated measurement of the radicle elongation rate [10]. Obviously, these alternative tests should be validated by a comparison with the results of traditional vigor tests for each species as requested by ISTA rules [5].

\section{FROM GERMINATING SEED 2-D IMAGES TO SURFACE 3-D MODELING AND POINT TRACKING}

Human visualization recognizes only three color sensors which are associated with long (Red, R), medium (Green, G) and short (Blue, B) wavelengths of light [15]. All visible colors can be represented by varying combinations of these primaries, as defined by a numeric range of RGB color space ranging from 255-255-255, for the so-called 'white' color, to 0-0-0 for the so-called 'black' color. Alternative color models have been proposed, and each color combination can be converted to the other [15]. In a previous work we reported a scheme of digital seed imaging processing by RGB value computation to obtain a 3-D simulation model of seed surface or a 2-D histogram of RGB data distribution [8]. Typically, smooth and simple surfaces, as resulting from a quick design using the most popular software programmes, such as Computer-Aided Design (CAD) or Computer-Aided Modeling (CAM), can be rendered in a 3-D model using subtle undulations and present textures on the real-world object. Scanning object image results in the collection of surface measurements which can be described as a mathematical function $z=f(x, y)$, where $z$ is the object depth for an $(x, y)$ location in the image plane [16]. In the case of digital image of crop seeds, we have used ImageJ software package (free download at: http://rsb.info.nih.gov/ij/ ) to modify 2-D images in a $3-D$ visualization mode which can approximate the 3-D original format of the seed. The 3-D surface plot plug-in of the software created a family of combinations of color, scale, rotation, perspective, smoothing, and different drawing modes. As an example, choosing appropriate parameters and the drawing mode of line fitted or mesh surface, it is possible convert a 2-D image of a radish seed captured by a full color CCD-camera to corresponding 3-D simulated models (Fig. 2).

The line fit mode of 3-D rendering of 2-D data extracted from a seed digital image generated a surface plot in which a series of 3-D histograms are displayed on the plane delimited by $\mathrm{X}$ and $\mathrm{Y}$ axes, with the height ( $\mathrm{Z}$ axis) due to the amplification of each pixel [17]. By changing the modality of smoothing and $\mathrm{Z}$ axis elevation, the mesh mode can enhance surface features, such as seed coat rugosity and color density distribution, disease and ruptures, which can be determined quantitatively using appropriate algorithms.

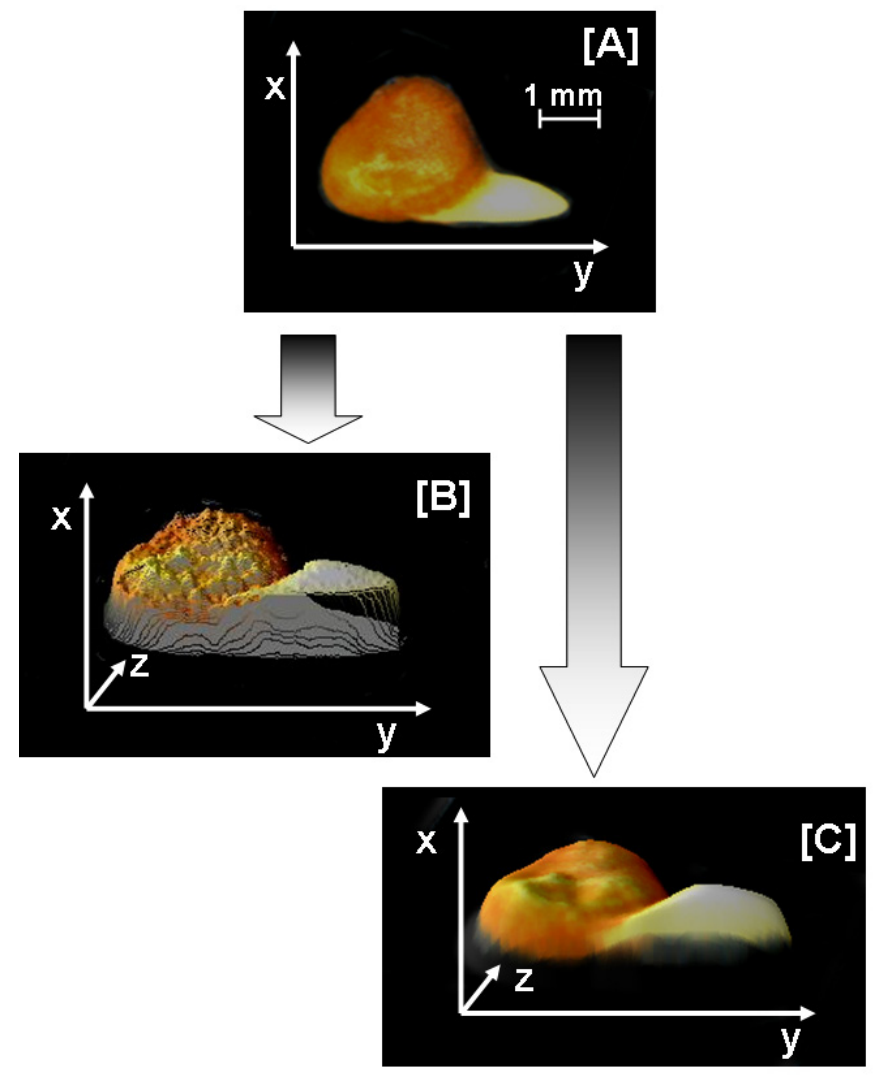

Fig. (2). Computer imaging of $21 \mathrm{~h}$ imbibed radish seed: A, 2-D image; $\mathbf{B}$, line mode of 3-D surface plot image; $\mathbf{C}$, mesh mode of 3D surface plot image. The simulation of 3-D images have been obtained using the interactive 3-D plotter plug-in of ImageJ software package.

More recently, a new technology has been developed known as dynamic speckle, or bio-speckle, an optical phenomenon occurring when biological tissues are illuminated by laser light. Features of seed tissue image, acquired by a CCD-camera, can be amplified and assessed by a specific image analysis software. The technique is not destructive and has been applied to test bean (Phaseolus vulgaris L.) seed viability and the presence of fungi colonies on the seed coat $[18,19]$. An alternative technique is the digital X-ray imaging, which was used to evaluate the effects of mechanical damages on seed quality of maize (Zea mays L.) [20]. X-rays photographs can be processed by an image analysis software to determine internal and external damages, proving a new non destructive technique for comparison of mechanical damages and seedling anomalies.

An interesting field of application of computer-assisted image analysis is that of seed growth intended as spatial expansion of a living organizm. Prior to the advent of computer technology, the velocity profiles of the extruded root tip from the seed coat were studied by marking (e.g., with ink or graphite) and imaging (e.g., with a series of photographs) over time [21]. Despite the basis of the measurement approach stayed the same, the photographs and the manual measurement of a point position on the studied object image were replaced by sequential time-lapse digital images automatically captured and by algorithms based on 
image sequence analysis in a 2-D velocity field, as reported by van der Weele et al. [22]. In the case of a germinating radish seed the variation in color space should be taken into account quite uniform as displayed on the seed surface prior to radicle protrusion from the seed coat, but changing in two different regions of color density distribution when the root tip emergence occurs. Seed growth can be studied as a continuous model involving two modules (i.e., the grain and the root tip) which change dimensions and color histogram patterns. One way of comprehensively describing the performance of an imbibing seed is by introducing parameters which can define the velocities at which determined points move relative to a fixed coordinate system, as discussed by Prusinkiewicz and his colleagues in defining models of more complex plant growth [23]. In germinating radish seed (Fig. 3, on the left) this can be represented by a vector connecting three points: $\mathrm{A}$, marking the left border of the seed image contour, C, marking the right border of the seed image contour and $\mathrm{B}$, which is the intersection point between the vector A-C and the contour of the seed grain (i.e., seed surface less the root tip surface). Once the vector is known, the way a region will change dimensions and color space distribution at time step can be computed (Fig. 3, on the right). Starting from $17 \mathrm{~h}$ imbibition, the vector A-C delimits the distance of the extreme boarders of the seed, with $\mathrm{B}=\mathrm{C}$. At $18 \mathrm{~h}$, when the root tip is emerging from the seed coat, a drop in Red and Green color primaries, and less visible for the Blue one, divides the color space of the grain from that of the root tip surface, corresponding to $\mathrm{B}$ point spatial position on the vector A-C. The trend of color patterns is confirmed at 19 and $20 \mathrm{~h}$, when the root tip length measures 1.2 and $2.4 \mathrm{~mm}$ respectively, and the distance B-C corresponding to elongation increase can be easily calculated by appropriate software algorithms. The distance A-B did not change significantly, suggesting the completion of grain sweling phase (i.e., phase I and II of the triphasic phase of water uptake). It can be concluded that to capture change in both modules of a germinating seed, each module has to be treated as a region that grows separately. This model of growth is obviously simplified and can not be applied to study a plant structure (e.g., branch or root systems) which includes the continuous change in module number to generate a more complex living shape than a germinating seed does [23].

\section{A LINK BETWEEN THE GERMINATION GEOMETRY CHANGES AND THE BIOLOGICAL SWITCH TO GERMINATION}

The characterization of biological and physical processes in growth models is usually based on the description of the geometric structure as a continuous process. In the last ten years 3-D measurements have developed into approaches to allow reconstruction of geometric structure of more common crop plants [24]. Modeling is based mainly on L-system or similar programmes, designed to simulate the 3-D architecture of higher plants [25]. L-systems were initially used to model the evolution of a set of cells [26], but they progressively gained in versatility and graphical capability to be a powerful tool to model the growth and the structure of plants. The development of geometric and mechanical patterns is determined by genes. Morphogenic studies on whole plants or their parts have been conducted to integrate experimental data into a regulatory network of 11 genes that control the shoot branching pattern and switch to flowering in Arabidopsis [27], or in more complex Antirrhinum (snapdragon) flower structure [1]. In addition, the concept of functional-structural modeling has been extensively developed in illustrating the model of root growth coupled with the assimilate transport, or the model of water transport in trees or that of radiant energy transfer in a scene of thousands plants, as extensively reviewed by Prusinkiewicz [28].
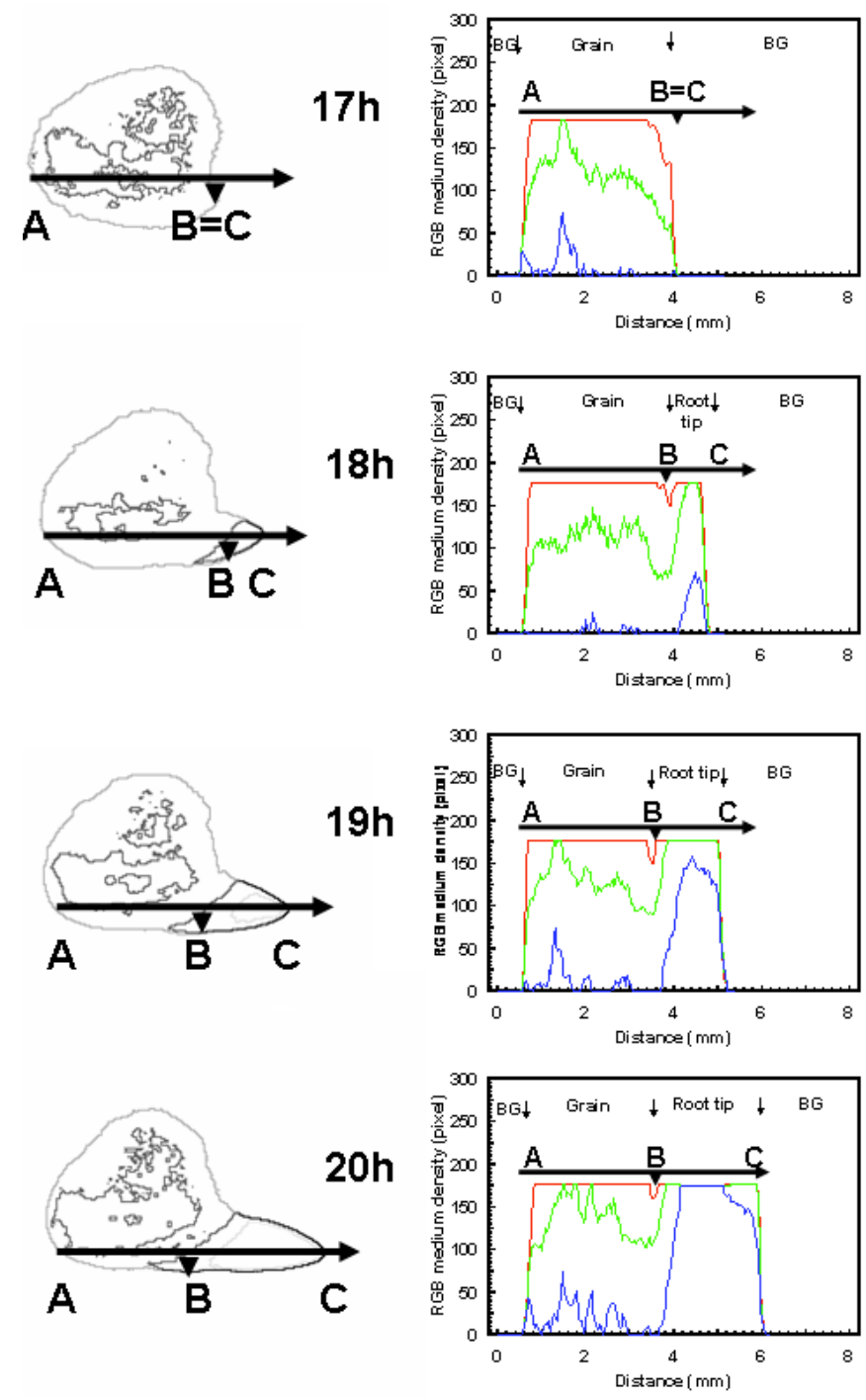

Fig. (3). Silhouette of radish seeds at different times of imbibition at $25^{\circ} \mathrm{C}$ (on the left) where two arbitrary points, $\mathbf{A}$ and $\mathbf{C}$, are fixed along the axis connecting the extreme points of seed surface contour. On the same axis, B marks the point of the seed external contour where the root tip extrusion occurs, and corresponds to point $\mathbf{C}$ prior to the radicle emergence. Color distribution histograms (on the left) reports Red-Green-Blue (RGB) color component distribution along the mentioned axis; $\mathrm{BG}$, background. Seed silhouette and RGB density histogram data were obtained by Image-Pro Plus v.6.1 software package (Media-Cybernetics, USA).

In the case of germination process, 2-D extracted data from a seed image may describe two main growth discrete modules, the first deriving from the physical sweling stage 
and, the second from the active radicle protrusion and elongation, both characterizing the geometry of seed germination. Genetic control mechanisms regulating the initiation of germination includes a checkpoint where GAinduced germination genes involved in GA synthesis and GA response control the switching to germinative pathway. This genetic flow of information has been extensively illustrated in Arabidopsis and Brassica napus by DNA sequencing, microarray quantitative measurements of RNA levels of hundred of genes simultaneously, and the expression levels of proteins and their post-translational modification using 2D gel electrophoresis atlas of thousands of polypeptides [29, 30]. In pure physiological terms, radicle extension through the structures surrounding the embryo is the event that terminates germination and marks the start of seedling growth. Extension of the radicle is a turgor-driven process, possibly due to negative osmotic potential of the radicle cells, or to cell wall loosening sustained by xyloglucancleaving and $\beta$-mannase enzymes and structural proteins (i.e., expansions) which have the ability to disrupt the hydrogen bonds between cell wall polymers, or to the seed tissues surrounding the radicle which weaken, thus allowing the root tip to elongate $[2,31]$.

Despite the complexity of biological process leading to extrusion of radicle tip, which marks the 'visible germination', time-lapse sequence of seed images and their analysis by computer technology can represent a valid support to physiology, biochemistry and molecular understanding seed germination pattern. The design of new algorithms devoted to complex growth indices, as well as the possibility to image and analyze a single seed performance within a large seed population under different environmental conditions, make the image analysis technology more adaptable in the study of seed germination biology associated with other approaches, as shown in Fig. (4). The ultimate goal of systems biology applied to plant research is to generate a model to describe processes across all levels of biological organization, including data integration, system modeling, and generation of hypothesis in designing new experiments [32]. In the case of seed germination, a more integrated network of approaches is expected which can satisfy the versatility of each knowledge model, its predicting potential, and its association with seed production and basic research demand. In this context, a particular interest is arising the enhancement of the preservation and the utilization of germplasm in a seed-gene bank. Automation and robotics for measuring seed viability and seedling growth should be addressed to determine the link between seed longevity and vigor and to realize image-based databases.

Besides these aspects of seed germination quality, it is generally known that higher plants produce heterogeneous seeds with respect to the extent of dormancy, dispersion and persistence within a soil-bank. Heterogeneity depends on seed development affected by several factors due to genetic and physiological components, the pattern being not uniform in any given population [33]. The physical differences within a seed population due to size, shape, density, surface texture and color, can be tested by mechanical or visual grading or by the property to be separated by gravity or air-stream or liquid fluctuation [34]. One of the physical characteristics most used as a marker of seed quality is seed size.
Controversial results have been reported on the influence of seed size on longevity, germination performance and mechanical injury, due to type of mechanical procedure used [35]. Removing non mature seeds or with mechanical damages by image analysis techniques allows the selection of high quality seeds, resulting in an increase of germination rate uniformity and healthy seeds, and, so, limiting the number of dormant seeds [4]. The new sorting methodology may be useful in seed technology for their automated, reliable and non-invasive procedures, and addressed to meet the request of seed industry in improving trade value and production yield. It should be desirable that the distribution of information among plant breeders and seed scientists can lead to define non-invasive bio-sensors to substitute tedious and subjective tasks usually employed in testing seed quality.

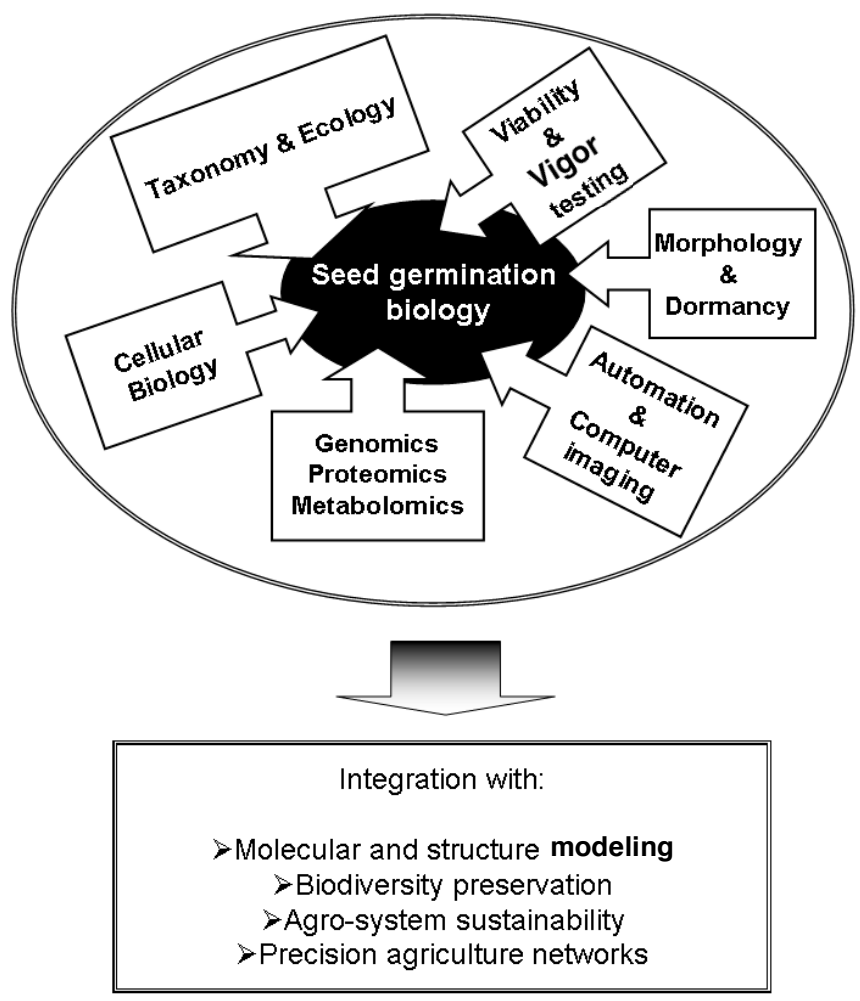

Fig. (4). Scheme of seed germination biology system integrating several approach systems with molecular and structure modeling, biodiversity preservation, agro-system sustainability, and precision agriculture networks.

\section{CONCLUSIONS}

Seed germination has intrigued the human activity since the late Neolithic age, because of practical reasons becoming a milestone in the 'agriculture framework' [36]. Actually a variety of computational techniques have been proposed to improve the visualization of seed germination data. These techniques include the capture of seed digital images and their processing, discerning and measuring shape, size and color space components, and establishing their modification and connectivity over germination time. In addition, it is noteworthy to mention the human-computer interaction, that might be required to localize seed points for image segmentation, modeling, or tracking. 
The integration of the different 'layers' of approach in seed biology and testing may lead to the implementation of databases and networks interrelating seed producers and growers, seed researchers and analysts. One aspect regarding the development of an appropriate technology is the control of crop quality assessment methods which needs automated equipments and data (2- and 3-D images and numerical extracted measures) processing and transferring to other computers networks. These efforts are comprised in the information technology [37], the main basis of precision agriculture, in terms of providing new systems frameworks of analysis and diagnostic methodologies for the maintenance of agricultural systems.

\section{REFERENCES}

[1] Coen E, Roland-Lagan A-G, Matthews M, Bangham A, Prusinkiewicz P. The genetics of geometry. Proc Natl Acad Sci USA 2004; 101: 4728-35.

[2] Bewley JD. Seed germination and dormancy. Plant Cell 1997; 9: 1055-66.

[3] Prusinkiewicz P, Rolland-lagan A-G. Modeling plant morphogenesis. Curr Opin Plant Biol 2006; 9: 83-8.

[4] Dell'Aquila A. Towards new computer imaging techniques to seed quality testing and sorting. Seed Sci Technol 2007; 38: 519-38.

[5] ISTA. International rules for seed testing. International Seed Testing Association 2005.

[6] AOSA. Rules for testing seeds. Association of Official Seed Analysts 2000.

[7] Cox S. Information technology: the global key to precision agriculture and sustainability. Comp Elect Agric 2002; 36: 93-111.

[8] Dell'Aquila A. Digital imaging information technology applied to seed germination testing. A review. Agron Sustain Dev 2008; 28: $1-9$.

[9] Dell'Aquila A, van Eck JW, van der Heijden GWAM. The application of image analysis in monitoring the imbibition provcess of white cabbage (Brassica oleracea L.) seeds. Seed Sci Res 2000; 10: $163-9$

[10] Dell'Aquila A. Application of a computer-aided image analysis system to evaluate seed germination under different environmental conditions. Ital J Agron 2004; 8: 225-9.

[11] Dell'Aquila A. The use of image analysis to monitor the germination of seeds of broccoli (Brassica oleracea) and radish (Raphanus sativus). Ann Appl Biol 2005; 146: 545-50.

[12] Sako Y, McDonald MB, Fujimura K, Evans AF, Bennet MA. A system for automated seed vigor assessment. Seed Sci Technol 2001; 29: 625-36

[13] Hoffmaster AL, Fujimura K, McDonald MB, Bennet MA. An automated system for vigor testing three-day-old soybean seedlings. Seed Sci Technol 2003; 31: 701-13.

[14] Xu L, Fujimura K, McDonald MB. Automatic separation opf overlapping seedlings by network optimization. Seed Sci Technol 2007; 35: 337-50.

[15] Fairchild MD. Color appearance models. MA, USA: AddisonWesley, Reading 1998.
[16] Willis A, Speicher J, Cooper DB. Rapid prototyping objects from scanned measurement data. Im Vis Comp 2007; 25: 1174-84.

[17] Barthel KU. 3D-data representation with Image J; http://rsb.info.nih.gov/ij/plugins/surface-plot-3d.html

[18] Braga R, dal Fabbro IM, Borem FM, et al. Assessment of seed viabilità by laser speckle techniques. Biosyst Eng 2003; 86: 297304.

[19] Braga R, Rabelo GF, Granato LR, et al. Detection of fungi in beans by the laser biospeckle technique. Biosyst Eng 2005; 91: 465-9.

[20] Cicero SM, van der Heijden GWAM, van der Burg WJ, Bino RJ. Evaluation of mechanical damage in seeds of maize (Zea mais L.) by X-ray and digital imaging. Seed Sci Technol 1998; 26: 603-12.

[21] Ericson RO. Modeling of plant growth. Ann Rev Plant Physiol 1976; 27: 407-34.

[22] van der Weele CM, Jiang HS, Palaniappan KK, Ivanov VB, Palaniappan K, Baskin TI. A new algorithm for computational image analysis of deformable motion at high spatial and temporal resolution applied to root growth. Roughly uniform elongation in the meristem and also after an abrupt acceleration, in the elongation zone. Plant Physiol 2003; 132: 1138-48.

[23] Coen E, Rolland-Lagan A-G, Matthews M, Bangham A, Prusinkiewicz P. The genetics of geometry. Proc Natl Acad Sci USA 2004; 101: 4728-35.

[24] Fournier C, Andrieu B. A 3D architectural and process-based model of maize development. Ann Bot 1998; 81: 233-50.

[25] Prusinkiewicz P, Lindermayer A. The algorithms beauty of plants. New York: Springer-Verlag 1990.

[26] Lindermayer A. Mathematicals models for cellular interaction in development. J Theor Biol 1968; 18: 280-315.

[27] Mendoza L, Alvarez-Buylla ER. Dynamics of the genetic regulatory network for Arabidopsis tailiana flower morphogenesis. J Theor Biol 1998; 193: 307-19.

[28] Prusinkiewicz P. Modeling plant growth and development. Curr Opin Plant Biol 2004; 7 : 79-83.

[29] Gallardo K, Job C, Grrot SPC, et al. Proteomic analysis of Arabidopsis seed germination and priming. Plant Physiol 2001; 126: 835-48.

[30] van der Geest AHM. Seed genomics: germinating opportunities. Seed Sci Res 2002; 12:145-53.

[31] Bewley JD. Breaking down the walls-a role for endo- $\beta$-mannase in release from seed dormancy? Trends Plant Sci 1997; 2: 464-9.

[32] Katagiri F. Attacking complex problems with the power of systems biology. Plant Physiol 2003; 132: 417-9.

[33] Matilla A, Gallardo M, Puga-Hermida M-I. Structural, physiological and molecular aspects of heterogeneity in seeds: a review. Seed Sci Res 2005; 15: 63-76.

[34] Halmer P. Enhancing seed performance. Seed technology and its biological basis. Sheffield: Sheffield Academic Press 2000; pp. 257-86.

[35] Hampton JC, Rahman MM, Mwakangwale MG, Hill MJ. Does seed weight explain vigor differences in seeds from different pod positions on the plant? Seed Sci Technol 2004; 33: 499-503.

[36] Evenari M. Seed physiology: its history from antiquity to the beginning of the $20^{\text {th }}$ century. Bot Rev 1984; 50:119-41.

[37] Cox S. Information technology: the global key to precision agriculture and sustainability. Comp Electr Agric 2002; 36: 93-111. 